\title{
Estilo personal de terapeutas que trabajan con pacientes severamente perturbados: un estudio cuantitativo y cualitativo
}

\begin{abstract}
RESUMEN: Estudio comparativo entre terapeutas de distintas orientaciones teóricas que asisten o no a pacientes severamente perturbados, con el objetivo de evaluar las características del estilo personal consideradas óptimas. PALABRAS CLAVE:

Estilo personal del terapeuta, investigación empírica, pacientes severamente perturbados.
\end{abstract}

\begin{abstract}
SUMMARY: A comparison between the personal style of the therapists from different theoretical orientations that usually work or not with severely disturbed patients, with the aim of evaluating the optimal characteristics of the personal style.

KEY WORDS:

Personal style of the therapist, empirical research, severely disturbed patients.
\end{abstract}

\section{Introducción}

En las últimas décadas la investigación empírica ha proporcionado hallazgos significativos sobre las variables que participan en el proceso y el resultado de la psicoterapia, a través de estudios centrados en las características del paciente, del terapeuta y de la relación terapéutica $(1,2,3)$. Particularmente, con respecto a la investigación sobre las variables del terapeuta, los principales desarrollos se han dirigido a diferenciarlas en variables de rasgo frente a variables de estado (rasgos: observables e inferidos; estados: observables e inferidos). Los estados observables incluyen variables como: entrenamiento del terapeuta, experiencia, estilo terapéutico interpersonal (estilos interactivos recíprocos, patrones verbales de interacción en psicoterapia, patrones de comunicación multicanal y no verbales). Dentro de la misma categoría se encuentran los métodos de tratamiento utilizados: manualizados, supervisión, adherencia/sintonía y habilidad/competencia; y las clases de intervenciones: directividad/no directividad del terapeuta, intervenciones orientadas al insight frente a los síntomas, emotivas vs. de soporte, intensidad del tratamiento, autodeclaraciones del terapeuta (1).

La evolución de la investigación en psicoterapia ha avanzado progresivamente, por un lado, hacia la determinación de cuáles son los terapeutas más adecuados para qué pacientes y en qué condiciones específicas y, por otro lado, se ha ocupado de establecer principios generales de la psicoterapia. Este fenómeno se pone en evidencia en los esfuerzos clínicos y de investigación de algunos programas como el de Beutler \& Harwood (4) que han estudiado la relación entre variables del paciente, los tipos de intervención del terapeuta y su relación con los resultados de la psicoterapia. 
En esta línea, el grupo de investigación en psicoterapia liderado por Fernández-Álvarez en Argentina, ha operacionalizado el constructo multidimensional Estilo Personal del Terapeuta (EPT), que es definido como: «El conjunto de características que cada terapeuta pone en juego en cada acto de psicoterapia, modelando los atributos fundamentales de dicho acto». El EPT alude a las condiciones singulares que conducen a un terapeuta a operar de un modo particular en su desempeño profesional y condiciona, por lo tanto, los alcances del modelo teórico empleado y las técnicas específicas aplicadas en cada tratamiento (5).

El EPT es un componente presente en toda psicoterapia, que influye en la marcha de cada proceso terapéutico e incide sobre los resultados, aunque en diferente grado según el tipo de paciente, la patología y el contexto de aplicación específico. Está constituido por un conjunto de funciones que en la práctica se llevan a cabo de manera integrada y expresan las disposiciones, rasgos y actitudes que todo terapeuta pone en evidencia en su ejercicio profesional. Estas funciones terapéuticas no pretenden abarcar exhaustivamente el carácter multidimensional del estilo personal del terapeuta, ni explicar la totalidad de las acciones que se llevan a cabo en la terapia. Se proponen, en cambio, como un criterio útil para delimitar los principales ejes que componen el acto psicoterapéutico $(5,6)$.

Se han realizado varios estudios sobre algunas variables que lo modulan, teniendo en cuenta la incidencia que probablemente el EPT ejerce sobre el proceso y el resultado de la psicoterapia:

a) Relaciones entre el estilo personal del terapeuta y el perfil personal del paciente

En un proyecto multicéntrico Buenos Aires-Barcelona se investigó la compatibilidad entre el estilo personal del terapeuta y las características personales del paciente, específicamente la interacción de las variables resistencia y estilo de afrontamiento del paciente con el EPT, y su efecto en distintos momentos del proceso psicoterapéutico, tanto en la alianza terapéutica como en las condiciones sintomáticas (7).

También se realizó la adaptación al español del S.T.S. (Selección Sistemática de Tratamiento) de Larry Beutler, y se concluyó que las interacciones terapeutacliente son moduladores importantes de los resultados de la terapia, mostrando que aquellos pacientes más resistentes respondieron significativamente mejor al tratamiento interactuando con terapeutas más espontáneos operativamente; y que los pacientes con un estilo de afrontamiento predominantemente externalizador respondieron significativamente mejor a la terapia interactuando con terapeutas más pautados (8). 
ORIGINALES Y REVISIONES

b) El efecto modulador que la orientación teórica y los años de experiencia clínica ejercen sobre el estilo personal del terapeuta.

Se llevaron a cabo estudios comparativos sobre la influencia de la orientación teórico-técnica y los años de experiencia, en las funciones que evalúa el EPT. Se intentó establecer en qué medida estas variables se comportan como moduladoras de las funciones que componen el EPT. Los resultados mostraron diferencias estadísticamente significativas en su composición para distintos grupos de psicoterapeutas (psicoanalíticos, cognitivos e integrativos). También se constató en este estudio que los años de experiencia tienen un efecto modulador parcial sobre la evolución de las distintas funciones del EPT. Además, el instrumento se mostró sensible para discriminar a los terapeutas en las funciones instruccional y atencional respecto a la duración predominante de los tratamientos que realizan $(9,10,11)$.

Otra investigación, en esta misma dirección, estuvo orientada a establecer si existen características diferenciales en los estilos terapéuticos en función de las poblaciones clínicas asistidas. Se ha estudiado el EPT de terapeutas que trabajan en Unidades de Cuidados Intensivos Neonatales (UCIN): los terapeutas que se desempeñan en UCIN se describieron significativamente más flexibles en la función instruccional que sus controles. En cuanto a la orientación de los terapeutas de la muestra bajo estudio (psicoanalistas/terapeutas integrativos) que trabajan con esta población clínica, no se hallaron diferencias significativas en ninguna de las funciones del EPT. Hecho que sí se produce al considerar terapeutas que trabajan en otras áreas de intervención, dónde se encontraron diferencias en las funciones expresiva, atencional y operativa; los psicoanalistas se describieron menos pautados, de atención más abierta y más distantes emocionalmente (12).

En la misma línea, el objetivo de este trabajo es investigar las características del estilo personal de terapeutas de distintas orientaciones teóricas que trabajan con pacientes severamente perturbados.

Los pacientes severamente perturbados se pueden considerar pacientes difíciles no sólo por el tipo de patología que presentan, sino por las dificultades en su disponibilidad para el tratamiento en general, y para el establecimiento de la alianza terapéutica en particular. Esta última condición constituye un requisito fundamental para que el tratamiento con estos pacientes sea eficaz $(13,14)$. Se trata de pacientes en los cuales la organización personal se encuentra psicopatológicamente comprometida, tanto en lo que hace a su funcionamiento mental como a la presencia de déficit funcionales para adaptarse satisfactoriamente a contextos relacionales y sociales.

Según Perris y McGorry (15), los tratamientos psicoterapéuticos para los trastornos severos han sido inapropiados, inefectivos y demasiado arcaicos e infle- 
xibles para las necesidades de estos pacientes. A pesar de los esfuerzos de FrommReichmann, Arieti, Kohut y otros por adaptar los abordajes psicoanalíticos tradicionales a los grupos de pacientes más severos; estos intentos, aislados de otros abordajes de tratamiento, finalmente no tuvieron el éxito esperado. Las terapias comportamentales tuvieron una historia similar.

Los ensayos clínicos controlados tampoco pudieron probar la efectividad de las psicoterapias dinámicas especializadas, ni del abordaje psicológico centrado en la persona para el tratamiento de las psicosis o los trastornos de la personalidad.

En la última década aparecen abordajes para la enfermedad mental grave. Actualmente existen varios ensayos clínicos aleatorizados que probaron la efectividad de la psicoterapia cognitiva para el tratamiento de la psicosis y los trastornos de personalidad.

Las propuestas cognitivas proponen que la base para la mejoría y el progreso del paciente severamente perturbado es una relación estable y saludable que se nutre activamente (15). Chadwick, Birchwood y Trower (16) plantean que el principal desafío del terapeuta al trabajar con personas que presentan delirios o alucinaciones es el problema de involucrar al paciente en la terapia. En este sentido proponen algunas modificaciones del setting y de los aspectos operativo-expresivos del terapeuta, esto es, sacrificar algunos de los límites básicos y principios de la psicoterapia al trabajar con estos pacientes, así como la utilidad de sesiones más cortas y más frecuentes. Además es a menudo más productivo estructurar las sesiones, evitar silencios prolongados y no presionar al paciente para que revele ideas delirantes.

Vallis (17) y Perris (18) enfatizan la necesidad de flexibilidad del terapeuta para el trabajo con pacientes severamente perturbados, tanto en la conceptualización como en las intervenciones concretas.

Desde los desarrollos psicodinámicos, Kohut (19) plantea que los terapeutas que trabajan con estos pacientes según su modelo teórico, suelen tener una actitud más relajada y despreocupada. Tienen menos recelos en cuanto a ponerse emocionalmente a disposición de sus pacientes si la necesidad lo exige y, por lo común, se conducen con menos reservas y prevenciones que la mayoría de los analistas, particularmente frente a los pacientes con trastornos narcisistas de la personalidad, aunque no ha llevado a cabo trabajos de investigación al respecto. Otros autores como Clarkin, Yeomans y Kernberg (20) también han realizado aportaciones a la psicoterapia de los pacientes severos.

La investigación que se presenta explora si existen características estilísticas diferenciales en las dimensiones del EPT de terapeutas que asisten a pacientes severamente perturbados, con especial atención al grado de involucración y distancia emocional. Específicamente se realizó un estudio comparativo entre tera- 
ORIGINALES Y REVISIONES

peutas que trabajan con pacientes severamente perturbados (pacientes internados y /o pacientes que presentan cuadros psicopatológicos agudos) y un grupo control conformado por terapeutas que no atienden habitualmente ese tipo de pacientes.

\section{Método. Muestra}

La muestra fue intencional, y se obtuvo en centros psiquiátricos públicos y privados de la ciudad de Buenos Aires y de Mar del Plata. Participaron en el estudio un total de 60 terapeutas, 30 dedicados a la clínica de pacientes severamente perturbados (edad media 43,36 $\pm 8,81 ; 16,73 \pm 9,09$ años de experiencia promedio; $66,7 \%$ mujeres; $40 \%$ psicoanalistas y $60 \%$ cognitivos e integrativos) y 30 terapeutas que formaron parte de la muestra control (edad media 43,66 $\pm 8,21 ; 16,76 \pm$ 8,77 años de experiencia promedio; $90 \%$ mujeres; $40 \%$ psicoanalistas y $60 \%$ cognitivos e integrativos). Se consideraron integrativos los terapeutas que utilizaban recursos teórico-técnicos pertenecientes a más de un enfoque tradicional (21).

Los grupos se homologaron en las variables socio-descriptivas de edad, orientación teórico-técnica y años de experiencia que acreditaban como psicoterapeutas. Participaron significativamente más mujeres que hombres en el estudio realizado $\left(x^{2}=4,81 ; p<.05\right)$.

En esta investigación la categoría de «pacientes severamente perturbados» incluyó cuadros psiquiátricos agudos (psicosis agudas y esquizofrenia), trastorno depresivo mayor, trastorno bipolar, trastornos de personalidad severos (predominantemente borderline), demencias y adicciones, condición que se estableció como criterio para la conformación de los grupos.

\section{Instrumentos}

Registro de datos básicos: se diseñó con el fin de obtener de forma sistematizada información socio-descriptiva y de la actividad clínica de los terapeutas.

Encuesta para terapeutas que trabajan con pacientes severos (ETPS): compuesta por siete preguntas abiertas con formato autoadministrado que indagan sobre: a) razones o motivos que tienen los terapeutas para trabajar con pacientes severos; b) descriptores y características de la involucración con pacientes severos; c) descriptores y características de la proximidad emocional con pacientes severos (Apéndice A).

Cuestionario del Estilo Personal del Terapeuta. EPT-C (6): se trata de un instrumento autoadministrado de 36 ítems, diseñado para evaluar un conjunto de fun- 
ciones terapéuticas propuestas en la definición del constructo y que caracterizarían el EPT. El inventario se responde en función del grado de acuerdo (Likert 1-7) para cada uno de los reactivos propuestos, donde el puntaje «1» significa un total desacuerdo con el enunciado, y el puntaje «7» significa un total acuerdo. Finalmente, se obtienen puntajes para cada una de las funciones que pueden ser expresadas en un perfil del EPT. Estas dimensiones son:

Instruccional: (puntajes más altos indican mayor rigidez). Se trata de las conductas que pone en marcha un terapeuta para establecer y regular el dispositivo de la terapia. Explora la flexibilidad-rigidez con que cada terapeuta tiende a operar con sus pacientes. Ejemplos de ítems: «Procuro que los pacientes se adecuen al formato habitual de mi trabajo»; «Suelo atender pacientes fuera del ámbito del consultorio».

Expresiva: (mayores puntajes aluden a mayor proximidad emocional). Son las acciones que lleva a cabo el terapeuta para sostener la comunicación emocional con el paciente. Explora la distancia-proximidad en dicha comunicación. Ejemplos de ítems: «Los verdaderos cambios se producen en el curso de sesiones con un clima emocional intenso»; «Evito revelar a mis pacientes mis estados emotivos».

De involucración: (a puntuaciones más altas, mayor grado) es el conjunto de conductas explícitas e implícitas vinculadas con el compromiso que experimenta el terapeuta frente a su tarea en general y, especialmente, frente a sus pacientes. Ejemplos de ítems: «Tengo en mente los problemas de los pacientes más allá de las sesiones»; «Mantengo un bajo nivel de involucración con los pacientes para actuar con más objetividad».

Atencional: (puntuaciones más altas describen atención más focalizada). Se refiere al modo en que el terapeuta focaliza su atención para obtener la información necesaria de sus pacientes. Ejemplos de ítems: «Suelo mantener mi escucha más bien abierta y receptiva más que concentrada y focalizada»; «Me gusta sentirme sorprendido por el material del paciente sin tener ideas previas».

Operativa: (puntajes altos equivalen a terapeutas más pautados). Hace referencia a las acciones directamente vinculadas con las intervenciones terapéuticas específicas. Ejemplos de esto son el grado de influencia o de directividad con que el terapeuta opera, o la preferencia por utilizar procedimientos más pautados o más espontáneos (por ejemplo: «Los tratamientos pautados me merecen escasa valoración»; «Como terapeuta prefiero indicar a los pacientes qué debe hacerse en cada sesión»). Un listado completo de los ítems se muestra en el apéndice B.

El EPT-C mostró condiciones psicométricas satisfactorias con respecto a su confiabilidad tanto para la consistencia interna como para la estabilidad temporal (alpha de Cronbach; test-retest). Y el procedimiento de análisis factorial realizado (componentes principales, rotación Varimax y normalización Kaiser) determinó 
una composición de cuatro factores que sugirió una validez teórica adecuada para las dimensiones propuestas, y explicó en su conjunto el 40.1\% de la varianza (6).

\section{Procedimiento y análisis}

Todos los terapeutas cumplimentaron de forma anónima y voluntaria el registro de datos básicos y el cuestionario EPT. Sólo a los terapeutas de pacientes severos se les administró complementariamente la ETPS.

Se realizaron análisis (pruebas t y U de Mann-Whitney) para determinar: a) si el tipo de pacientes asistidos (severos vs. controles) modulaba el EPT; b) si existían diferencias en el EPT de los terapeutas que atienden pacientes considerados severos vs. controles según la orientación de pertenencia (cognitiva e integrativa vs. psicoanálisis); y c) si existían diferencias en el EPT de los terapeutas cognitivos e integrativos vs. psicoanalistas según la población clínica asistida (severos vs. controles). En el análisis estadístico de los datos aportados en b) y en c) se consideraron conjuntamente los terapeutas cognitivos e integrativos en un mismo grupo.

Para el análisis de la información cuantitativa se utilizó el software SPSS 13.0, y para el análisis cualitativo el software Atlas.Ti versión 4.2.

\section{Resultados}

\section{EPT y población clínica}

En primer lugar se compararon las valoraciones dadas al EPT-C por los terapeutas que asisten a pacientes severos vs. terapeutas que no atienden a este tipo de pacientes, tomados en conjunto (sin distinción por orientación).

Tabla 1

Diferencias en el EPT-C según población clínica asistida

\begin{tabular}{|c|c|c|c|}
\hline $\begin{array}{c}\text { EPT-C } \\
\text { Población clínica }\end{array}$ & $\begin{array}{c}\text { Severos } \\
(\mathrm{n}=30)\end{array}$ & $\begin{array}{c}\text { Controles } \\
(\mathrm{n}=30)\end{array}$ & $\mathrm{t}$ \\
\hline Instruccional & $3.68 \pm 1.03$ & $3.99 \pm .95$ & $\mathrm{n} . \mathrm{s}$. \\
\hline Atencional & $2.84 \pm .98$ & $2.86 \pm 1.14$ & n.s. \\
\hline Expresiva & $3.50 \pm 1.14$ & $4.04 \pm 1.10$ & $-1.83^{*}$ \\
\hline Operativa & $4.00 \pm 1.23$ & $2.96 \pm 1.02$ & $3.53^{* * *}$ \\
\hline Involucración & $3.22 \pm 1.12$ & $4.26 \pm 1.35$ & $-3.23^{* *}$ \\
\hline
\end{tabular}

n.s.: no significativa; $* \mathrm{p}<0.05 ; * * \mathrm{p}<0.01 ; * * * \mathrm{p}<0.001$ 
Los terapeutas de pacientes severos se consideraron significativamente más distantes en la comunicación emocional con sus pacientes, $\mathrm{t}(58)=-1.83 ; \mathrm{p}<.05$, con una menor involucración, $\mathrm{t}(58)=-3.23 ; \mathrm{p}<.01$, y más pautados operativamente, $\mathrm{t}(58)=3.53 ; \mathrm{p}<.001$, que aquellos terapeutas que asisten a pacientes considerados no severos. No fueron significativas las diferencias en las funciones instruccional y atencional del EPT para las poblaciones clínicas consideradas (Tabla 1).

2. EPT, orientación teórica y población clínica

Se analizaron los datos para cada orientación teórica. En el caso de los terapeutas cognitivos e integrativos, aquellos cuya actividad clínica estaba basada en la atención de pacientes severamente perturbados, se describieron en su estilo personal como significativamente menos involucrados y más pautados, al ser comparados con los terapeutas de la misma orientación que atendían pacientes con trastornos considerados no severos. También resultaron más distantes emocionalmente, más flexibles y menos focalizados aunque esta tendencia no fue significativa. (Tabla 2a).

Tabla 2a

Diferencias en el EPT-C de terapeutas que atienden distintas poblaciones clínicas según la orientación teórico-técnica

\begin{tabular}{|l|l|l|l|l|l|l|}
\hline ORIENTACIÓN & \multicolumn{3}{|c|}{ Cognitivo-integrativa } & \multicolumn{3}{c|}{ Psicoanalistas } \\
\hline $\begin{array}{l}\text { EPT-C } \\
(\mathrm{n}=18)\end{array}$ & $\begin{array}{l}\text { Severos } \\
(\mathrm{n}=18)\end{array}$ & Controles & $\mathrm{U}$ & $\begin{array}{l}\text { Severos } \\
(\mathrm{n}=12)\end{array}$ & $\begin{array}{l}\text { Controles } \\
(\mathrm{n}=12)\end{array}$ & $\mathrm{U}$ \\
\hline Instruccional & $3.76 \pm .81$ & $4.07 \pm .73$ & n.s. & $3.55 \pm 1.32$ & $3.86 \pm 1.24$ & n.s. \\
\hline Atencional & $3.22 \pm 1.00$ & $3.39 \pm .83$ & n.s. & $2.26 \pm .64$ & $2.06 \pm 1.10$ & n.s. \\
\hline Expresiva & $3.99 \pm 1.02$ & $4.22 \pm .68$ & n.s. & $2.77 \pm .92$ & $3.76 \pm 1.53$ & $43.00^{*}$ \\
\hline Operativa & $4.06 \pm 1.23$ & $3.42 \pm .96$ & $109.50^{*}$ & $3.9 \pm 1.27$ & $2.27 \pm .67$ & $21.00^{* *}$ \\
\hline Involucración & $3.52 \pm .92$ & $4.43 \pm 1.05$ & $84.00^{* *}$ & $2.78 \pm 1.29$ & $4.01 \pm 1.72$ & $42.50^{*}$ \\
\hline
\end{tabular}

n.s.: no significativa; $* \mathrm{p}<0.05 ; * * \mathrm{p}<0.01 ; * * * \mathrm{p}<0.001$

Cuando el análisis de las diferencias en el EPT se centró en los psicoanalistas, éstos se describieron con una mayor distancia emocional, menos involucrados y más pautados con los pacientes severamente perturbados que los psicoanalistas que centraban su actividad clínica en poblaciones menos severas. También los terapeutas psicoanalíticos que asistían a pacientes severos se mostraron algo más flexibles en el establecimiento y mantenimiento del setting terapéutico, y con una atención más focalizada aunque sin alcanzar la significación estadística. (Tabla 2a). 
Se analizaron las diferencias entre terapeutas cognitivos e integrativos vs. psicoanalistas para las poblaciones clínicas consideradas. Los terapeutas de pacientes severamente perturbados que se adscribían a una orientación cognitiva e integrativa se describieron como más focalizados atencionalmente, más cercanos emocionalmente, y con una mayor involucración que sus pares psicoanalistas. Pero no se diferenciaron significativamente los grupos profesionales en las funciones instruccional y operativa. (Tabla $2 b$ ).

Tabla $2 b$

Diferencias en el EPT-C de distintas orientaciones según población clínica asistida

\begin{tabular}{|c|c|c|c|c|c|c|}
\hline \multirow{2}{*}{$\begin{array}{c}\text { Población } \\
\text { clínica } \\
\text { EPT-C }\end{array}$} & \multicolumn{3}{|c|}{ Severos } & \multicolumn{3}{|c|}{ Controles } \\
\hline & $\begin{array}{l}\text { Cogn- } \\
\text { integ. } \\
(n=18)\end{array}$ & $\begin{array}{l}\text { Psicoan. } \\
(\mathrm{n}=12)\end{array}$ & $\mathrm{U}$ & $\begin{array}{l}\text { Cogn- } \\
\text { integ. } \\
(n=18)\end{array}$ & $\begin{array}{l}\text { Psicoan. } \\
(\mathrm{n}=12)\end{array}$ & $\mathrm{U}$ \\
\hline Instruccional & $3.76 \pm .81$ & $3.55 \pm 1.32$ & n.s. & $4.07 \pm .73$ & $3.86 \pm 1.24$ & n.s. \\
\hline Atencional & $3.22 \pm 1.00$ & $2.26 \pm .64$ & $42.00 * *$ & $3.39 \pm .83$ & $2.06 \pm 1.10$ & $33.5 * * *$ \\
\hline Expresiva & $3.99 \pm 1.02$ & $2.77 \pm .92$ & $43.50 * *$ & $4.22 \pm .68$ & $3.76 \pm 1.53$ & n.s. \\
\hline Operativa & $4.06 \pm 1.23$ & $3.90 \pm 1.27$ & n.s. & $3.42 \pm .96$ & $2.27 \pm .67$ & $35.0^{* * *}$ \\
\hline Involucración & $3.52 \pm .92$ & $2.78 \pm 1.29$ & $63.00 *$ & $4.43 \pm 1.05$ & $4.01 \pm 1.72$ & n.s. \\
\hline
\end{tabular}

n.s.: no significativa; $* \mathrm{p}<0.05 ; * * \mathrm{p}<0.01 ; * * \mathrm{p}<0.001$

Particularmente los terapeutas cognitivos e integrativos se describieron más pautados y con una atención más focalizada que los psicoanalistas cuando la población clínica asistida era de pacientes menos severos. Sin embargo, los grupos no presentaron diferencias estadísticamente significativas en las funciones instruccional, expresiva y de involucración en el grupo control.

3. Evaluación cualitativa a través de la ETPS de las dimensiones: «involucración» $\mathrm{y}$ «expresiva» de terapeutas que asisten a pacientes severos

A continuación se describen las respuestas de los terapeutas que trabajaban con pacientes severos a la ETPS. El ítem 1 se utilizó como criterio de agrupabilidad (población clínica predominantemente asistida) y, por lo tanto, no se incluyó en el análisis. Las respuestas al ítem 2 permitieron operacionalizar la variable «pacientes severos». Esto fue descrito más arriba en el apartado Muestra. 
(Ítem 3) ¿Por qué razón o razones usted trabaja con este tipo de pacientes?

En este estudio el 53,3\% de los terapeutas manifestaron interés y motivación personal para asistir a pacientes severamente perturbados, hubo un 33,3\% que atribuyó su trabajo con esta población clínica a exigencias y razones institucionales y un 13,3\% combinó ambos tipos de razones.

(Ítem 4) ¿Cómo describiría el grado de involucración más conveniente del terapeuta?

Los terapeutas cuya motivación se liga a un tipo de atribución personal (interna) son los que mostraron una mayor tendencia a expresar altos grados de involucración. Esta involucración asume un carácter reflexivo y busca adecuarse a las características del paciente. Las expresiones que adopta toman como referente la consideración de aspectos contratransferenciales. Este segmento de terapeutas considera que su labor exige un alto grado de disponibilidad hacia el paciente. Ejemplos de respuestas son:

Alto nivel de involucración y disponibilidad (telefónica, entrevistas adicionales, etc.). Disposición a tolerar situaciones de alta carga emocional o de conflicto. Requiere mucha involucración, pensar diferentes alternativas fuera de las sesiones. A veces es necesario atenderlos por teléfono, lo que interfiere en la vida personal, sobre todo en momentos críticos.

Los terapeutas cuya motivación se liga a motivos institucionales (externa) tienden a circunscribir un mayor grado de involucración a situaciones críticas (riesgo de suicidio). Se muestran propensos a una relación con el paciente mucho más mediada por marcos teóricos de comprensión que por la referencia a sí mismos. El trabajo en equipo y la supervisión es considerado importante en casi todos los casos.

(Ítem 5) ¿Hay algún tipo de pacientes severos frente a los cuales decide asumir un tipo especial de involucración? ¿Por qué motivos?

La necesidad de considerar conveniente una involucración diferencial para determinado tipo de pacientes generó respuestas dispares. Ejemplos:

- «Sí, los pacientes con trastorno de personalidad tipo borderline. Por el tipo de sostén que necesitan basado en la confianza y soporte de sus desbordes».

- «Sí, por motivos inherentes a la patología del paciente».

- «En determinados casos donde el riesgo de suicidio es alto, a fin de lograr que el paciente evalúe otras opciones».

- «No. Induciría a cierta subjetividad inadecuada».

- «No, no creo que haya pacientes especiales». 
ORIGINALES Y REVISIONES

(Ítem 6) ¿Cómo describiría la proximidad emocional más conveniente del terapeuta?

Los mismos terapeutas que se muestran proclives a mayores niveles de involucración son quienes consideran importante prestar atención al grado óptimo de proximidad emocional. Por ese motivo sobre este plano mantienen un criterio selectivo, discriminando las características del paciente y el posicionamiento personal acorde:

- «Lo primero que intento es lograr empatía con el paciente».

- «No podría pensar en una distancia emocional óptima para todos los pacientes severos, puesto que se trata de un grupo muy heterogéneo. Evalúo la proximidad emocional en función de otros criterios».

- «No es posible plantearse una conducta emocional a priori. Esto se produce en situación, sin perder de vista que el terapeuta es una función».

Algunos tratan de vincularla a la comprensión del caso y las características del proceso:

- «Las emociones influyen pero no son decisivas. No creo conveniente revelar los estados afectivos del terapeuta pero sí tenerlos en cuenta para entender lo que le sucede al paciente. No es imprescindible para promover cambios terapéuticos una alta proximidad emocional».

- «La proximidad debe ser cálida y continente que genere un clima de confianza. Utilizo la contratransferencia en la comprensión del estado mental de los pacientes».

Un grupo menor se muestra refractario a asignarle valor o significación a esta dimensión en el proceso terapéutico:

- «No debe existir».

- «La abstinencia por parte del profesional».

(Ítem 7) ¿Hay algún tipo de pacientes severos frente a los cuales decide asumir un tipo especial de proximidad emocional? ¿Por qué motivos?

Un grupo predominante (el mismo que se muestra proclive a mayores niveles de involucración y que considera importante encontrar un grado óptimo de proximidad emocional) considera que determinados pacientes requieren un tipo particular de proximidad o distancia emocional:

- «Sí, es importante tener en cuenta los estados afectivos que generan algunos pacientes al terapeuta cuando ellos mismos no manifiestan sus afectos (no manifiestan angustia, tristeza, dolor, no hablan, etc.)». 
- «Si el paciente es actuador o manejador pongo más distancia».

- «Frente al desencadenamiento de un amor de transferencia histeriforme conviene adoptar una abstinencia amable».

- «En función del estilo de vinculación interpersonal del paciente y las modificaciones o cambios a implementar de acuerdo a los objetivos terapéuticos (mayor proximidad en momentos de crisis, mayor distancia en los períodos en que es necesario favorecer autonomía o mayor responsabilidad)».

En síntesis: aquellos terapeutas que manifestaron una motivación personal por atender a pacientes severos se describieron con una mayor involucración, una mayor consideración de la proximidad emocional, más disponibilidad hacia las necesidades del paciente y más consideración por los aspectos que hacen a la relación terapéutica dentro de la terapia, comparados con los que atendían a esta población clínica por exigencias institucionales de su lugar de trabajo.

Esta tendencia estuvo relacionada positivamente con las autodescripciones cuantitativas del estilo en las funciones expresiva y de involucración, obtenidas a través del EPT-C.

\section{Discusión}

Los resultados muestran diferencias significativas en el estilo personal de los terapeutas que asisten a pacientes severamente perturbados. Estas diferencias se manifestaron en tres de las funciones del EPT: expresiva, de involucración y operativa. En estos casos, los terapeutas valoraron una involucración significativa baja, una mayor distancia emocional y una mayor estructuración en los aspectos operativos de la psicoterapia. Aún es necesario establecer los efectos que estas características estilísticas tienen sobre el establecimiento y mantenimiento de la alianza terapéutica, aspecto central en el tratamiento de estos pacientes $(13,14)$ y sobre los resultados de la psicoterapia.

Podríamos interpretar esto como un mecanismo de protección de los terapeutas ante la mayor exigencia que plantea el trabajo con pacientes severos. Esto discrepa con muchas de las respuestas de los profesionales a la encuesta cualitativa. Probablemente porque debido a la mayor demanda de involucración que exigen los pacientes severos, los terapeutas tiendan a establecer una mayor distancia emocional y un menor grado de involucración como mecanismo compensatorio en la relación, independientemente de la valoración que hacen de lo que consideran adecuado u óptimo en el tratamiento de este tipo de pacientes. 
Sería importante investigar también la incidencia de este tema en la salud mental y en la calidad de vida de los terapeutas. Es interesante mencionar que los terapeutas que trabajan con pacientes severos se perciben en el nivel operativo, significativamente más pautados en todas las orientaciones teóricas incluidas en el estudio, algo esperable en función del tipo de tratamiento que llevan adelante (Tabla 2a). Sin embargo surgen algunas diferencias: los controles cognitivos se describen más involucrados que los terapeutas cognitivos que atienden pacientes severos; y esta diferencia es mucho más significativa entre los terapeutas cognitivos e integrativos que entre los psicoanalistas. Mientras que los cognitivos e integrativos no difieren significativamente en la función expresiva.

También es interesante mencionar que entre los psicoanalistas, los que trabajan con pacientes severos son notablemente más pautados, y esta diferencia es más significativa que entre los cognitivos. Esto podría explicarse por la mayor distancia existente entre la prescripción teórica con respecto a las intervenciones que presentan los psicoanalistas y las demandas reales que plantean los pacientes severos.

$\mathrm{Si}$ analizamos ahora las diferencias entre las orientaciones para cada población clínica (Tabla 2b) notamos que los cognitivos e integrativos son mucho más cercanos emocionalmente y más involucrados que los psicoanalistas, cuando trabajan con pacientes severos, a diferencia de lo que sucede en la muestra control donde se mantiene la tendencia, aunque sin alcanzar significación estadística. Es interesante mencionar la dimensión operativa: en el caso de los controles son mucho menos pautados los psicoanalistas que los cognitivo-integrativos (como su orientación teórica lo prescribe). Sin embargo estas diferencias se diluyen con los pacientes severos. También la dimensión atencional es significativa y marca nuevamente una diferencia: los terapeutas cognitivos e integrativos manifiestan una atención más focalizada que sus pares psicoanalistas, diferencia que se evidencia para ambas poblaciones clínicas. Como otros trabajos han sugerido, el tipo de pacientes atendidos también modula el EPT, así como la orientación teórica y los años de experiencia $(10,9,12)$.

En síntesis, dentro de la misma orientación, los terapeutas muestran diferencias de estilo, pero también la población clínica asistida pone en evidencia modos diferentes de abordaje según la orientación de los terapeutas.

Es necesario considerar estos resultados con cautela debido al tamaño de la muestra, y realizar estudios en muestras más amplias, con otras poblaciones clínicas y también con terapeutas de diferentes regiones geográficas.

Si bien los resultados obtenidos muestran la capacidad heurística del constructo EPT así como la sensibilidad del instrumento propuesto, por delante tenemos varios desafíos. La relevancia del constructo debería ser puesta a prueba a tra- 
vés de dos aspectos. Primero, su estabilidad temporal: los terapeutas deberían tener un estilo similar a través del tiempo en intervalos no muy largos, ya que se ha conceptualizado el EPT como estable en el tiempo, aunque no necesariamente constante. Puede mantenerse por largos períodos y puede sufrir cambios como resultado del entrenamiento recibido, por cambios en las condiciones de trabajo, y también debido a factores evolutivos u otras circunstancias de la vida del terapeuta (6). Esto se ha constatado a través de la confiabilidad test-retest del instrumento EPT-C.

Otro aspecto importante para investigar sería la consistencia transituacional del constructo. En este caso la consistencia del estilo terapéutico se expresaría por su estabilidad a través de diferentes tipos de pacientes. Está claro que los terapeutas no nos comportamos exactamente igual con todos nuestros pacientes, sin embargo debemos suponer que el estilo muestra un patrón de variabilidad y que este patrón mantiene cierta estabilidad temporal. Una propuesta análoga es la de los actuales modelos interaccionistas de la personalidad (22). Seguramente desde esta perspectiva la experiencia y la formación teóricoclínica van moldeando a los terapeutas así como las experiencias vitales van moldeando nuestra personalidad. Estamos trabajando en la búsqueda de la metodología más adecuada para detectar la existencia de esos patrones de variabilidad estilística.

\section{BIBLIOGRAFÍA}

(1) Beutler, L. E., y otros, «Therapist Variables», en LAMBerT, M. (ed.), Bergin and Garfield's Handbook of Psychotherapy and Behavior Change, EE.UU., Wiley, 2004, 5. a edición, pp. 227-306.

(2) ORlinsky, D. E.; Howard, K. I., «Process and Outcome in Psychotherapy», en Garfield, S. L. y Bergin, A. E. (eds.), Handbook of Psychotherapy and Behavior Change, Nueva York, Wiley, 1986, pp. 311-381.

(3) Orlinsky, D. E.; Grawe, K.; Parks, B. K., «Process and Outcome In Psychotherapy. Noch Einmal», en Bergin, A. B. y GArFIELD, S. L. (eds.), Handbook of Psychotherapy and Behavior Change, Nueva York, Wiley, 1994, 4. ${ }^{\mathrm{a}}$ ed., pp. 270-376.

(4) Beutler, L. E.; Harwood, T. M., Prescriptive Psychotherapy, Nueva York, Oxford University, 2000.

(5) FernáNDEZ-Álvarez, H.; García, F., «El estilo personal del terapeuta: Inventario para su evaluación», en GRIL, S., y otros (eds.), Investigación en Psicoterapia, Pelotas (Brasil), Educat, 1998, pp. 76-84.

(6) FERnÁNDEZ-ÁlVAREZ, H., y otros, «Assessment Questionnaire on The Personal Style of the Therapist PST-Q», Clinical Psychology and Psychotherapy, 10, 2003 pp. 116-125.

(7) Corbella SAntomá, S., Compatibilidad entre el estilo personal del terapeuta y el perfil personal del paciente, TD, Facultat de Psicologia, Ciències de l'Educació i de l'Esport Blanquerna, Universitat Ramon Llull, Barcelona, 2002. 
(8) MaLIK, M. L., y otros, Which Therapist for Which Client? The Interaction of Therapist Variation and Client Characteristics in Affecting Rates of Change: An Effectiveness Study, Ponencia presentada en el «Annual Meeting of the Society for Psychotherapy Research», San Francisco, 2002.

(9) CastañeIras, C., y otros, Modulating Effect of Experience and Theoretical-Technical Orientation on the Personal Style of the Therapist, en preparación.

(10) CASTAÑEIRAS, C., y otros, The Influence of Experience and Theorical-Technical Orientation on the Personal Style of the Therapist, Ponencia presentada en el «Annual Meeting of the Society for Psychotherapy Research», Weimar, 2003.

(11) FERNÁNDEZ-ÁlVAREZ, H., y otros, Estilos personales e terapeutas psicoanalíticos, cognitivos e integrativos, Ponencia presentada en el «II Congresso Latinamericano de Pesquisa em Psicoanalise e Psicoterapia», Gramado, 2000.

(12) Vega, E., Perfil del psicoterapeuta neonatal, Tesis de Doctorado en Psicología, Facultad de Psicología, Universidad de Buenos Aires, 2004.

(13) Semerari, A. (ed.), Psicoterapia cognitiva del paciente grave, Bilbao, Desclée de Broker, 2002.

(14) Perris, C., Psicoterapia del paziente difficile, Lanciano, Métis, 1993.

(15) Perris, C.; McGorry, P. D. (eds.), Cognitive Psychotherapy of Psychotic and Personality Disorders. Handbook of Theory and Practice, Chichester, Wiley, 1998.

(16) Chadwick, P.; Birchwood, M.; Trower, P., Cognitive Therapy for Delusions, Voices and Paranoia, Gran Bretaña, Wiley, 1996.

(17) Vallis, T. M., «When the Going Gerts Tough: Cognitive Therapy for the Severely Disturbed», en Perris, C. y McGorry, P. D. (eds.), Cognitive Psychotherapy of Psychotic and Personality Disorders. Handbook of Theory and Practice, Chichester, Inglaterra, Wiley, 1998, pp. 37-62.

(18) Perris, H., «Less Common Therapeutic Strategies and Techniques in the Cognitive Psychotherapy of Severely Disturbed Patients», en Perris, C. y McGorry, P. D. (eds.), Cognitive Psychotherapy of Psychotic and Personality Disorders. Handbook of Theory and Practice, Chichester, Wiley, 1998, pp. 315-332.

(19) Kohut, H., ¿Cómo cura el análisis?, Buenos Aires, Paidós, 1984.

(20) Clarkin, J. F.; Yeomans, F. E.; Kernberg, O. F., Psychotherapy for Borderline Personality Disorders, Nueva York, Wiley, 1999.

(21) Fernández Álvarez, H., Fundamentos de un modelo integrativo en psicoterapia, Buenos Aires, Paidós, 1992.

(22) Mischel, W., «Toward an Integrative Science of the Person», en FISKE, S. T., y otros (eds.), Annual Review of Psychology, Vol. 55, Palo Alto, CA, Annual Reviews Inc, 2004, pp. 1-22. 
ORIGINALES Y REVISIONES

\section{Apéndice A}

\section{FUNDACION AIGLE}

Encuesta para terapeutas que trabajan con pacientes severos

N. ${ }^{\circ}$ de orden:

Fecha:

1) ¿Trabaja usted predominantemente con pacientes severos? SI $\square$

2) Si su respuesta es SI, ¿podría especificar con qué tipo de pacientes severos?

3) ¿Por qué razón o razones usted trabaja con ese tipo de pacientes?

Involucración

La labor clínica con pacientes severos requiere diferentes grados de involucración* por parte del terapeuta. Queremos conocer qué piensa usted sobre algunos aspectos de este tema.

4) ¿Cómo describiría el grado de involucración más conveniente del terapeuta en el tratamiento del paciente severo?

5) ¿Hay algún tipo de pacientes severos frente a los cuales decide asumir un tipo especial de involucración? ¿Por qué motivos?

Proximidad emocional:

Del mismo modo, los terapeutas mantienen diferentes grados de cercanía emocional** con sus pacientes. Queremos conocer qué piensa usted sobre algunos aspectos de este tema.

6) ¿Cómo describiría la proximidad emocional más conveniente del terapeuta en el tratamiento del paciente severo?

7) ¿Hay algún tipo de pacientes severos frente a los cuales decide asumir un tipo especial de proximidad o distancia emocional? ¿Por qué motivos?

* Por ejemplo: ¿cuánto tiene en mente los problemas de sus pacientes fuera de las sesiones?, ¿cuánto piensa en su trabajo en sus horas libres? ¿cuánto influye en su vida personal lo que le pasa a sus pacientes?

** Por ejemplo: ¿En qué medida usted cree que las emociones que le despierta el paciente son decisivas para el curso del tratamiento?, ¿le resulta útil revelar algunos de sus aspectos personales en las sesiones?, ¿piensa que una alta proximidad emocional con los pacientes es imprescindible para promover cambios terapéuticos?

Muchas gracias por su colaboración. 


\section{ORIGINALES Y REVISIONES}

\section{Apéndice B}

\section{EPT-C}

Total desacuerdo // 1-2-3-4-5-6-7\ Total acuerdo

01. Suelo mantener mi escucha más bien abierta y receptiva más que concentrada y focalizada.

02. Procuro que los pacientes se adecuen al formato habitual de mi trabajo.

03. Como terapeuta prefiero indicar a los pacientes qué debe hacerse en cada sesión.

04. Mantengo un bajo nivel de involucración con los pacientes para actuar con más objetividad.

05. Me resultan estimulantes los cambios en el encuadre.

06. Las emociones que me despierta el paciente son decisivas para el curso del tratamiento.

07. Me siento más inclinado a acompañar al paciente en su exploración que a señalarle los caminos a seguir.

08. Evito comunicarme a través de gestos y expresiones muy emotivas.

09. Tiendo a exigir cumplimiento estricto con los honorarios.

10. Los tratamientos pautados me merecen escasa valoración.

11. La expresión de las emociones es un poderoso instrumento de cambio.

12. Muchos cambios importantes en el curso de un tratamiento requieren que el terapeuta opere con un bajo tono emocional.

13. Fuera de las horas de trabajo dejo de pensar en los pacientes.

14. Los cambios de consultorio afectan negativamente los tratamientos.

15. Los verdaderos cambios se producen en el curso de sesiones con un clima emocional intenso.

16. Me considero un terapeuta que trabaja con un encuadre flexible.

17. Me resulta útil revelar algunos de mis aspectos personales en las sesiones.

18. Me gusta sentirme sorprendido por el material del paciente sin tener ideas previas.

19. Suelo atender pacientes fuera del ámbito del consultorio.

20. Las mejores intervenciones en un tratamiento surgen de modo espontáneo.

21. Lo que les pasa a mis pacientes tiene poca influencia en mi vida personal.

22. En mis intervenciones soy predominantemente directivo.

23. Pienso bastante en mi trabajo, aun en mis horas libres.

24. Evito revelar a mis pacientes mis estados emotivos.

25. Puedo planear un tratamiento en su totalidad desde que se inicia.

26. La distancia emocional con los pacientes favorece los procesos de cambio.

27. Nunca modifico la duración de las sesiones, a menos que sea imprescindible.

28. Si algo me irrita durante una sesión puedo expresarlo.

29. Una alta proximidad emocional con los pacientes es imprescindible para promover cambios terapéuticos. 
30. Prefiero saber de antemano a qué cosas debo prestar atención en las sesiones.

31. Prefiero los tratamientos donde están programados todos los pasos a seguir.

32. Me interesa trabajar con pacientes que presentan problemas focalizados.

33. Procuro dirigir mi atención a la totalidad de lo que pasa en las sesiones.

34. Tengo en mente los problemas de los pacientes más allá de las sesiones.

35. Soy bastante laxo con los horarios.

36. Desde el comienzo de las sesiones me dispongo a dejar flotar mi atención.

* Verónica Rial, licenciada en Psicología; Claudia Castañeiras, doctora en Psicología; Fernando García, doctor en Psicología; Beatriz Gómez, licenciada en Psicología; Héctor Fernández-Álvarez, doctor en Psicología. Todos de la Fundación Aiglé, Buenos Aires (Argentina).

Correspondencia: Verónica Rial. Fundación Aiglé. Virrey Olaguer y Feliú. 2679 CP C1426EBE. Ciudad Autónoma de Buenos Aires. Argentina.

fundacion@ aigle.org.ar Fax: 0054114784 3563. Tel.: 00541147813897. 